\title{
Voice Controlled Wheelchair
}

\author{
M. S. Arsha ${ }^{1 *}$, A. Remya Raj ${ }^{2}$, S. R. Pooja ${ }^{3}$, Rugma Manoj ${ }^{4}$, S. A. Sabitha ${ }^{5}$, Shimi Mohan ${ }^{6}$ \\ ${ }^{1,2,3,4}$ Student, Department of Electrical \& Electronics Engineering, Musaliar College of Engineering, \\ Trivandrum, India \\ ${ }^{5}$ Assistant Professor, Department of Electrical \& Electronics Engineering, Musaliar College of Engineering, \\ Trivandrum, India \\ ${ }^{6}$ Associate Professor, Department of Electrical \& Electronics Engineering, Musaliar College of Engineering, \\ Trivandrum, India \\ *Corresponding author: arshams@ieee.org
}

\begin{abstract}
This paper is a design of Voice Controlled Wheel Chair for people who has any physical illness. Here Arduino, microcontroller and geetech voice recognition module are used to support the motion of the wheelchair. In order to provide the battery level, a battery level indicator is also provided. Based upon the direction specified in the commands, the Arduino will drive the 2 motors. People those who has disabilities with their hands, foot and lower body are unable to perform tasks on regular basis. So, there are many applications which help handicapped person to perform their tasks. The aim of this system to help people who cannot move properly without help others due to any physical illness or disabilities. Speech recognition technology will provide a new way of human interaction with machine.
\end{abstract}

Keywords: Arduino, Battery level indicator, Ultrasonic sensor, Voice recognition module.

\section{Introduction}

A wheelchair is used when it is impossible to walk due to physical illness or disabilities. There are various kinds of wheelchairs which meet the various needs of their users.

Several studies have resulted that for independent mobility to all the disabled human. The motive is to help such people to live an independent life without depending on others. The wheelchair is navigated in the four directions [1]. By using the voice recognition module, the user can control the movement of the model by sending voice commands such as forward, reverse, left, right and stop. In order to provide the level of battery, a battery level indicator is also provided [2].

Generally, the communication port is the parallel port which takes the commands given to the wheel chair in the form of electrical signals [3]. Four wheels are used in the wheel chair for proper balancing. The movement of wheels is controlled by car wiper motors which are attached to the wheel chair.

The microcontroller based control systems are presented for disabled persons in [4].

\section{Proposed System}

The speech recognition block is having three sub modules which are Voice capture module, Voice customization module and Voice recognition module. Fig. 1 shows the block diagram of our proposed system. The Voice customization is used for training the kit to receive voice commands signals in which we are going to customize 5 commands which can lead directions of the wheelchair such as FRONT, BACKWARD, RIGHT, LEFT and STOP.

Then it converts the received commands into binary codes consisted with its frequency of the speech command which are getting to be compared with predefined command stored in the microcontroller [5]. In our project, it is used to detect obstacles and calculate the distance from the wheelchair and switch off the system immediately. For providing a battery level indication, an indicator is also provided.

The difference between the existing systems and proposed system is that we take wheel chair from hospitals and we implement voice recognition circuits and finally we hand over it back to the hospital, through this procedure we can hand reduce cost of making a new wheel chair and also we can reduce the overall cost. Two wheels located on the left side of the wheelchair are controlled by one motor and the wheels on the right side are controlled by the second motor.

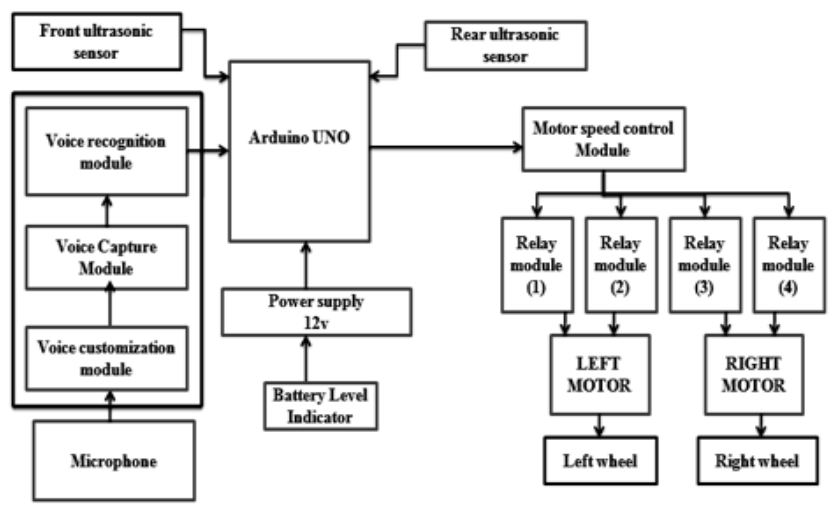

Fig. 1. Block diagram

\section{Components}

\section{A. Geetech voice recognition module}

Technology has developed an intelligent man-machine interface technique which facilitates computers or machines or 
robots to be operated using the voice commands of human without using any input systems, such as keyboards or mouse. Voice recognition is a method that facilitates natural and [6] convenient human-machine interface using the voice recognition module. It extracts and analyses voice features of human delivered to a machine or computer through the microphone.

Working Principle of voice recognition module is that HM2007 (fig. 2) is a single chip CMOS voice recognition module.

1) Features of voice recognition module

- Voltage:4.5-5.5V

- Current: $<40 \mathrm{~mA}$

- Digital Interface: 5V TTL level UART interface

- Analog Interface: 3.5mm mono-channel microphone connector + microphone pin interface

- Size: $30 \mathrm{~mm} \times 47.5 \mathrm{~mm}$

- Recognition accuracy: 99\% (under ideal environment)

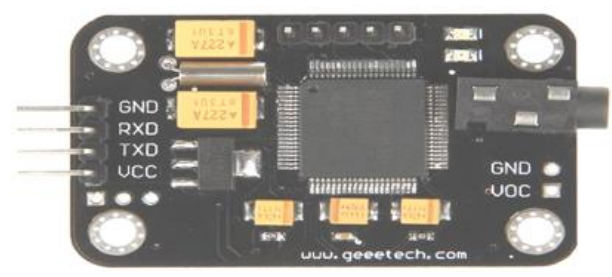

Fig. 2. Geetech voice recognition module

\section{B. Arduino}

The Uno has the difference that it does not use the FTDI USB-to-serial driver chip. No additional programmer is required for programming board.

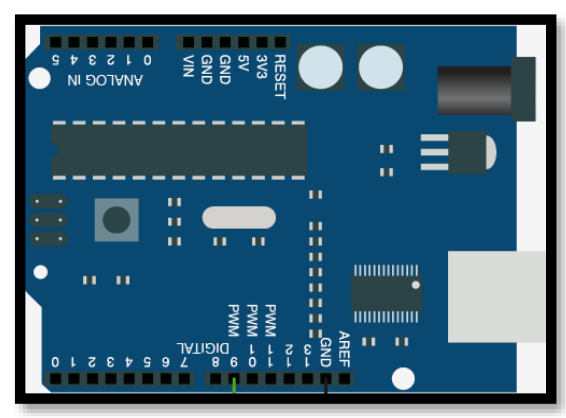

Fig. 3. Arduino

\section{1) Technical specifications}

- Microcontroller: AT Mega 328

- Operating voltage: $5 \mathrm{~V}$

- Input voltage (Recommended):

- 7-12 V

- Input voltage (Limits): 6-20 V

- Digital I/O pins: 14

- Analog input pin :6

- Flash memory :32 KB of which .5KB used by boot loader
- $\quad$ SRAM :2KB

- $\quad$ EEPROM : $1 \mathrm{~KB}$

- Clock Speed :16 MHz

\section{Relay H-Bridge}

To drive DC motors with different voltages and currents we can design a h-bridge with a relay board. This control logic is really smart, a short circuit will never happen, even during a transition state. For control a dc motor we need an h-bridge.it is the only way to drive motor forward, stop and reverse. Each pin of individual motor is connected to a relay and if the logic status is 1 it is connected to positive power if logical status is 0 it connected to ground. The term $\mathrm{H}$ Bridge is the typical graphical representation of the circuit. In simple words, the term $\mathrm{H}$ Bridge is derived from the flow of current in the circuit which you will understand completely with the help of the following example. An H bridge can be made using four switches, MOSFET's or Relays.

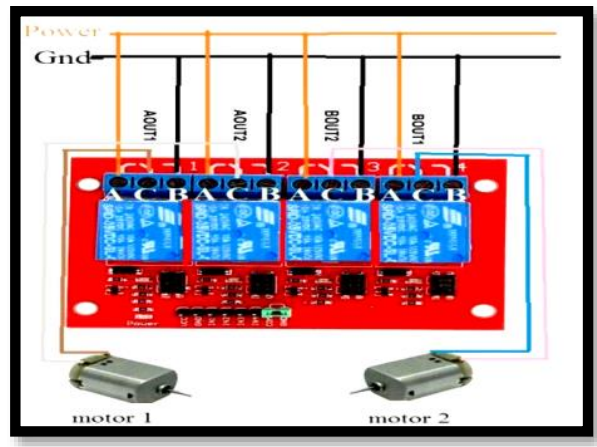

Fig. 4. Relay H-Bridge

\section{Wiper motor}

In order to move wiper motor it needs a power source. The different power sources are the batteries, voltages, current (minimum of $1.6 \mathrm{amps}$ at $70 \mathrm{rpm} ; 1 \mathrm{amps}$ at $41 \mathrm{rpm}$ ), computer batteries (12volts output) and other battery supplies that do not exceed the limit of 12 volts otherwise the motor is bound to overheat. In this project we use 2 wiper motors of $12 \mathrm{v}$.

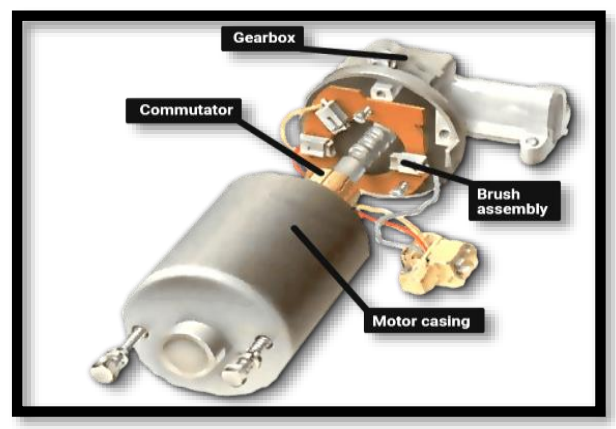

Fig. 5. Wiper motor

\section{E. Ultrasonic Sensor}

Ultrasonic sensors work by emitting sound waves at frequency too high for human beings to hear. They then wait for the sound to get reflected back, calculating. Ultrasound is 
reliable in any lighting environment and can be used either inside or outside. Ultrasonic sensors can handle collision avoiding mechanism for a robot, and being move often, as long as it isn't too fast.

These sensors are widely used and they can be implemented in water level sensing, drone applications and sensing cars at your local drive-thru restaurant or bank. Ultrasonic rangefinders are commonly used to detect a collision.

Distance $=$ product of Time and Speed of Sound in Air (340 $\mathrm{m} / \mathrm{s})) / 2$

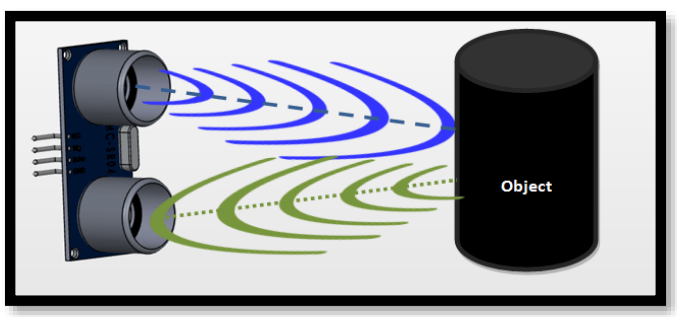

Fig. 6. Ultrasonic sensor

\section{1) Advantages}

- Can operate in many environment.

- Ultrasonic sensors work in smoke filled environment.

\section{F. Battery level indicator}

The heart of our battery level indicator circuit is LM3914 IC. This IC takes input voltage and drives 10 LED's linearly according to the input analog voltage. In this circuit LED's (D1D10) displays the capacity of the battery display mode. This mode is chosen by the external switch sw1 which is connected to 9th pin of IC. 6th \& 7th pins of IC are connected to ground through a resistor. This resistor controls the contrast of LED's. Here resistor R3 and POT RV1 forms potential divider circuit connection. Here pot RV1 is used for calibration of circuit. There is no need of any external power to this circuit.

The circuit is designed to monitor $10 \mathrm{~V}-15 \mathrm{~V}$ DC supply. The circuit will work even if the battery voltage is having $3 \mathrm{~V}$. The operating voltage of this IC is about $3 \mathrm{v}$ to $25 \mathrm{v}$ DC. LM3914 drives LED's, LCDs and vacuum fluorescents. The IC contains adjustable reference and accurate 10 -steps dividers. This IC can also act as sequencer in the indicator.

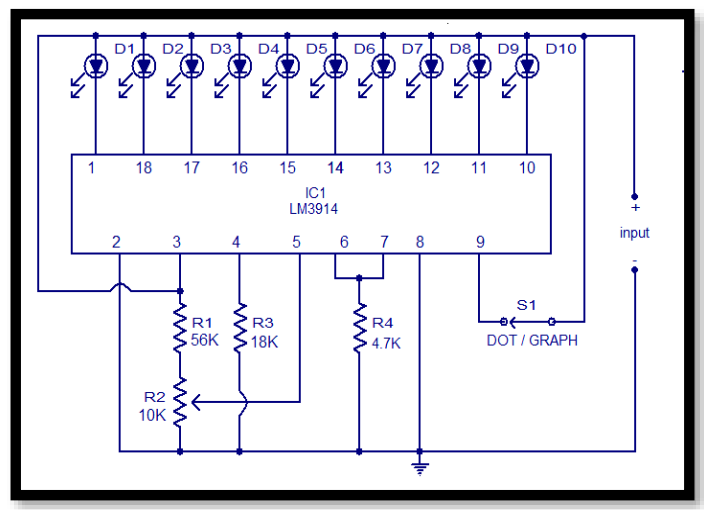

Fig. 7. Battery level indicator

\section{Circuit Diagram}

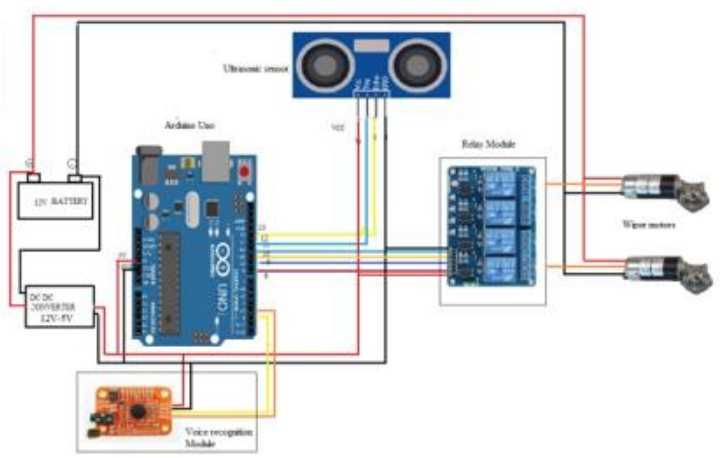

Fig. 8. Circuit diagram

\section{Working of Voice Controlled Wheelchair}

Input is taken through microphone. Speech signal is processed using with the help of speech recognition module and is transfer to the Arduino. Arduino converts these instructions into commands that can be recognized by the motors. This controls the movement and direction of wheel chair through motor driver. Arduino decides the operation of the two DC motors depending on the given instruction. Firstly, voice module is trained with four commands. After that the voice command is send by the person whose voice has being drained. The Arduino is used to check the signal associated with this command and compare it with the stored commands and performs the task related to this command.

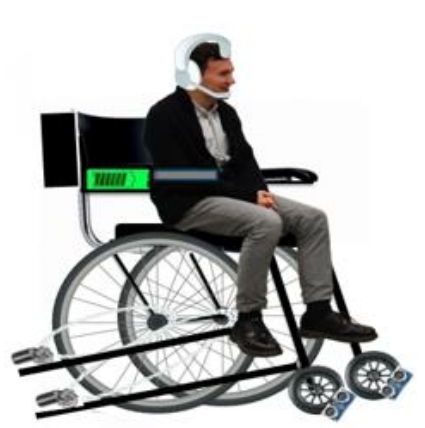

FORWARD

\section{BACKWARD}

\section{LEFT}

RIGHT

Fig. 9. Working

The wheel chair directions and movement possible are as given in fig. 10.

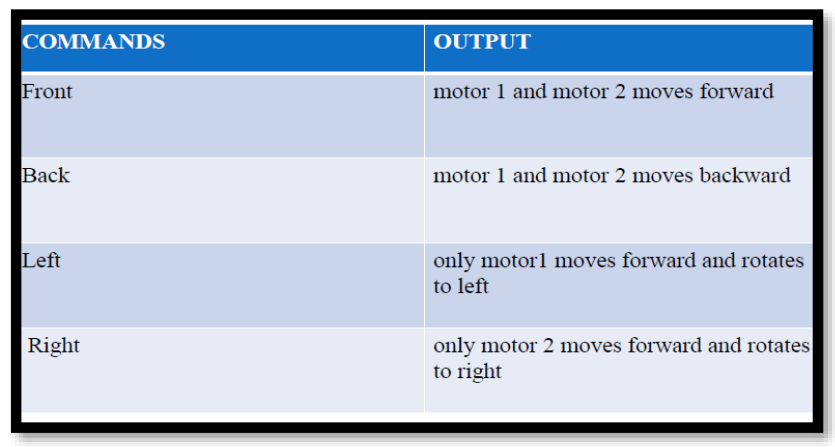

Fig. 10. Directions 
When the voice is detected, the wheel chair can be controlled to move in that direction by giving commands to the wheel chair, these commands are transferred using electrical signals which are used to drive the left or right motor of the wheel chair. There are two motors connected to the left and right wheels of the wheel chair, the electrical signals are transferred to these motors using some hardware ports, called the communication port is the parallel ports. Four wheels are used in the wheel chair for proper balancing. The movement of wheels is controlled by DC motors which are attached to the wheel chair. Two wheels located on the left side of the wheelchair are controlled by one motor and similarly the wheels on the right side are controlled by the other motor.

\section{Conclusion}

Voice controlled wheelchair has the key functionality of following voice commands. Along with the normal methods of operations such as joystick/keypad, a novel way of controlling the chair using a web application, will also be made available. This provides user the ability to control the wheelchair while sitting in some corner of the house.

The proposed model uses advanced hardware which not only processes the voice but also controls the motors, thus, reducing the number of hardware used and decreasing the cost.

However, there is also a disadvantage that it does not distinguish between a normal conversation and a command.

The problem of the recognition of commands by another person is minimized in a situation where the user is wearing a dynamic microphone with a narrow filed of sound, which also partially removes the background noise and enhances the change in the color of pronounced commands by the user. This does not have a larger effect on the quality of the recognition.

\section{Future Scope}

- Further advancement is possible by automatically charging battery with any renewable source of energy.

- Stair climbing mechanism can be implemented in future using voice command.

\section{References}

[1] J. Greichen, Arthur D, "Value based home automation for today's market”, IEEE Transactions on Consumer Electronics, vol. 38, no. 3, August 2002.

[2] Srinivasavaradhan L, Chandramouli G (2008), "Automated vehicles for physically and visually challenged", International Conference on Perspective Technologies and Methods in MEMS Design, MEMSTECH 2008, pp. 40-43

[3] Rajesh Kannan Megalingam, Ramesh Nammily Nair, Sai Manoj Prakhya, Mithun Mohan (2012), "GestBot' - A highly convenient locomotive solution for the elderly and physically challenged", IEEE Global Humanitarian Technology Conference, pp. 350-355.

[4] Kharka Bahadur Rai, Jeetendra Thakur, Nirmal Rai (2015), "Voice controlled wheelchair using Arduino", International Journal of Science, Technology \& Management, vol. 4, no. 6, pp. 6-13.

[5] Jinyi Long, Hongtao Wang, Yuanqing Li, Tianyou YuJiahuiPan, and Feng Li, "Speed of a Simulatedor Real Wheelchair", IEEE transactions on neural systems and rehabilitation engineering, 2012.

[6] Shubhangi P Patil, Seema G. Shirsikar, "Voice and Touchpad Operated Circuit for Wheel Chair".

[7] M. Senthil Sivakumar, Jaykishan Murji, Lightness D. Jacob, Frank Nyange, M. Banupriya, "Speech controlled automatic wheelchair", Pan African International Conference on Information Science, Computing and Telecommunications, 2013

[8] Shraddha Uddhav Khadilkar, Narendra Wagdanikar, "Android phone controlled Voice, Gesture and Touch screen operated Smart Wheelchair", IEEE, 2015.

[9] EasyVR 3 User Manual-VeeaR.” Available: http:// www.veear.eu/files/EasyVR-User-Manual.pdf. 